\title{
CHARACTERISTIC SUBMANIFOLDS OF FIXED POINT FREE INVOLUTIONS 1
}

\author{
ELLEN STONE
}

\begin{abstract}
According to López de Medrano, a manifold with a free involution is determined by its Browder-Livesay index and the normal cobordism class of its orbit space. Here we study the Browder-Livesay indices of some cobordant involutions, namely, the characteristic submanifolds of a fixed involution.
\end{abstract}

In [2] and [3], studying fixed point free involutions on homotopy spheres, W. Browder and G. R. Livesay defined an index $\sigma$ measuring the obstruction to finding an invariant embedded sphere of codimension 1. For a closed orientable manifold $M^{4 k-1}$ with a fixed point free involution $T$ which preserves orientation, the Browder-Livesay index $\sigma(T, M)$ is defined as the signature of a bilinear form on the homology of a characteristic submanifold; a characteristic submanifold $W$ is a closed, orientable submanifold of codimension 1 whose complement has two components $A$ and $B$ such that $B=T A$ and $\mathrm{Cl} A$ $\cap \mathrm{Cl} B=W$. Since characteristic submanifolds arise as $f^{-1} R P^{n-1}$ where $f: M / T \rightarrow R P^{n}$ (real projective space) is the classifying map of the $Z_{2}$ bundle $M \rightarrow M / T$, characteristic submanifolds exist and any two are equivariantly cobordant in $M \times I$. It follows that $\sigma$ does not depend on choice of $W$ [1].

However, if $M_{0}$ and $M_{1}$ are characteristic submanifolds of $\left(T, X^{4 k}\right)$, in general $\sigma M_{0} \neq \sigma M_{1}$; if $X$ is $S^{4 k}, \sigma M_{0}-\sigma M_{1}=2 \sigma\left(T \times 1 ; S \times I, M_{0}-M_{1}\right)$ [1] and if $M_{0}$ and $M_{1}$ are spheres, $\left(T, M_{0}\right)$ and $\left(T, M_{1}\right)$ are equivariantly diffeomorphic iff $\sigma M_{0}=\sigma M_{1}[2],[3]$.

Here we derive a formula for $\rho\left(M_{0}, M_{1}\right)=\sigma M_{0}-\sigma M_{1}-2 \sigma\left(X \times I, M_{0}-M_{1}\right)$ and show that it lies between $\pm \operatorname{dim} H_{2 k} X$ and does assume all these values. We also give an algebraic condition for a subspace of $H_{2 k} X$ to be $H_{2 k} A$ where $A \cap T A$ is a characteristic submanifold of $X$.

Received by the editors August 27, 1973.

AMS (MOS) subject classifications (1970). Primary 57E10.

Key words and phrases. Fixed point free involutions, Browder-Livesay index, equivariant cobordism, characteristic submanifold.

1 This is part of the author's doctoral thesis at Cornell University written under the direction of Professor G. R. Livesay. 
Throughout this paper, all manifolds are smooth or PL, compact and orientable, all involutions are smooth or PL and have no fixed poirits. Homology groups have rational coefficients, except where otherwise stated. $B$ will be used for any bilinear form on a subspace of $H_{2 k} N^{4 k}$ given by $B(x, y)=$ $x \cdot T y$, the intersection number of $x$ and $T y$ in $N$; the annihilator, under $B$, of a subspace $C$ will be denoted $\mathbb{Q} C$.

Suppose $S$ is an orientation reversing free involution on a compact manifold $Y^{4 k+1}$ :

Definition. A characteristic submanifold $N^{4 k}$ of $(S, Y)$ is an invariant submanifold of $Y$, meeting $\partial Y$ transversely (in $\partial N$ ) for which there are compact submanifolds $U$ and $V$ of $Y$ with $U \cup V=Y, U \cap V=N$ and $V=S U$. Then $\partial N$ will be a characteristic submanifold of $(S, \partial Y)$ splitting $\partial Y$ into $(U \cap \partial Y) \cup(V \cap \partial Y)$.

Definition. The Browder-Livesay index $\sigma(S ; Y, N)$ is the signature of the symmetric bilinear form $B(x, y)=x \cdot S y$ defined on $\operatorname{ker}\left(H_{2 k} N \rightarrow H_{2 k} U\right)$. In fact, given $\partial N$ this is independent of $N$.

Now suppose $\left(T, M^{4 k-1}\right)$ is a given characteristic submanifold of $\left(T, X^{4 k}\right)=(S \mid \partial Y, \partial Y)$. Then $M$ can be extended to a characteristic submanifold $N^{4 k}$ of $Y$, splitting $Y$ into $U \cup V$; let $A=U \cap X$ and $B=V \cap X$. Notice that $T / X$ reverses and $T / M$ preserves the orientation.

Suppose all maps in the diagram are induced by the inclusions. Let $K=$ $u^{-1} \operatorname{im} i \cap v^{-1} \operatorname{im} j \subseteq H_{2 k} N$, and $L=\operatorname{im} a \cap \operatorname{im} b \subseteq H_{2 k} Y$.

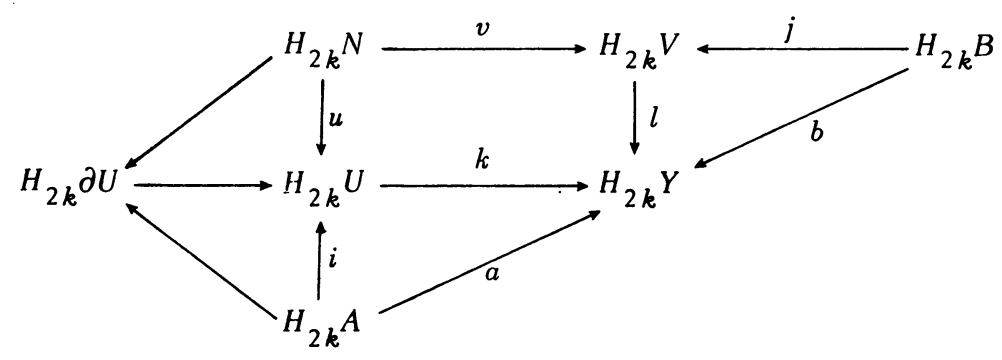

Define a bilinear form $\rho$ on $L$ as follows: if $x_{i}=a\left(s_{i}\right)=b\left(t_{i}\right), i=1,2$, set $\rho\left(x_{1}, x_{2}\right)=s_{1} \cdot T t_{2}$, where the intersection is taken in $X$.

Lemma. $\rho$ is well defined (i.e. is independent of the choices of $s_{i}$ and $t_{i}$ ) and is induced from the bilinear form $-B$ on $K$.

Proof. First we show there is an induced form. By the Mayer-Vietoris 
sequence for $Y$, the sequence

$$
0 \rightarrow \operatorname{ker} k u \cap K \rightarrow K \rightarrow L \rightarrow 0
$$

is exact. The kernel of $-B$ restricted to $K$ is $\mathbb{Q} K \cap K$, where

$$
\mathfrak{A} K=\mathfrak{A}\left(u^{-1} \operatorname{im} i \cap v^{-1} \operatorname{im} j\right)=\mathfrak{Q}\left(u^{-1} \operatorname{im} i\right)+\mathfrak{Q}\left(v^{-1} \operatorname{im} j\right) \supseteq \operatorname{ker} v+\operatorname{ker} u \text {, }
$$

since $\mathbb{Q}$ ker $v=u^{-1}$ im $i$. But by the Mayer-Vietoris sequence for $Y$, ker $u+$ ker $v=\operatorname{ker} k u$; therefore $\operatorname{ker} k u \cap K \subseteq \operatorname{ker}-B \mid K$, so that $-B$ induces a bilinear form on $L$.

To show that this induced form is $\rho$, let $x_{1}$ and $x_{2} \in L$, and pick $s_{i}$ and $t_{i}, i=1,2$, such that $x_{i}=a s_{i}=b t_{i}$; then there exist $m_{i} \in K$ such that $u m_{i}=i s_{i}$ and $v m_{i}=j t_{i}$. If $\rightarrow s_{1} \cdot T t_{2}=m_{1} \cdot S m_{2}$, since $x_{i}=k u m_{i}$, then $\rho$ is the induced form. In fact, since $\partial U=A \cup N$, intersection numbers are equal whether taken in $A$ and $N$ or in $\partial U$. Regarded as elements of $H_{2 k} \partial U,\left(s_{1}-m_{1}\right)$ and $T\left(t_{2}-m_{2}\right) \in \operatorname{ker}\left(H_{2 k} \partial U \rightarrow H_{2 k} U\right)$, hence their intersection number is 0 . Since $H_{2 k} A$ and $H_{2 k} N$ are orthogonal in $H_{2 k} \partial U, s_{1} \cdot T t_{2}+m_{1} \cdot S m_{2}=0$ i.e. $s_{1} \cdot T t_{2}=-m_{1} \cdot S m_{2}$. Therefore $\rho$ is the induced form and hence is well defined.

Theorem 1. $\sigma(T, M)=2 \sigma(S ; Y, N)$ - signature $\rho$ 。

Proof. Since $(T, M)=\partial(S, N)$ and $S$ has no fixed points, $\sigma(T, M)=$ signature $B$ on $H_{2 k} N$, where $B(x, y)=x \cdot S y$ [4], [5].

Notice that if $F$ is a bilinear form on a vector space $W$, and if $C$ is a subspace for which $C \subseteq \mathfrak{A}_{C}$, then signature $F=$ signature $F \mid \mathfrak{Q} C$. For by replacing $C$ by $C+\operatorname{ker} F$, we may assume $\operatorname{ker} F \subseteq C$; then $W$ can be written as $C \oplus D \oplus E$ where $\mathscr{A} C=C \oplus D$ and $\mathscr{C} D=C \oplus E$. Now $\operatorname{sig} F=\operatorname{sig} F \mid C \oplus E$ + $\operatorname{sig} F \mid D$, but since the annihilator of $C$ in $C \oplus E$ is $C$, sig $F \mid C \oplus E=0$, and since $C \subseteq \operatorname{ker} F \mid D \oplus C$, sig $F=\operatorname{sig} F \mid \mathbb{Q} C$.

By Poincaré duality, the kernel of $B$ on $H_{2 k} N$ is ker $p$ where $p: H_{2 k} N$ $\rightarrow H_{2 k} N, M$ is induced by the projection. Let $C=(\operatorname{ker} u+\operatorname{ker} p) \cap \mathbb{Q} \operatorname{ker} u$, then since $C \subseteq \mathfrak{Q} C$, sig $B=\operatorname{sig} B \mid \mathfrak{Q} C$; but $\mathfrak{Q} C=\mathfrak{Q}$ ker $u+\operatorname{ker} u$ where these two terms are orthogonal, so that

$$
\operatorname{sig} B=\operatorname{sig} B|\operatorname{ker} u+\operatorname{sig} B| \mathscr{Q} \operatorname{ker} u .
$$

Let $W=\mathfrak{Q} \operatorname{ker} u$ and let $J=\operatorname{ker} B \mid W$. Consider $D=\mathfrak{Q} \operatorname{ker} v \cap W$ and $E=\operatorname{ker} v+J$, where $E \subseteq W$ since $\operatorname{ker} v \subseteq \mathfrak{A}$ ker $u$. Then $\mathfrak{Q} E$ (in $W$ ) is $\mathfrak{Q}$ ker $v \cap \mathfrak{U}_{J} \cap W=D$ and since $E \supseteq J, E=\mathfrak{Q} \mathscr{Q} E=\mathscr{A} D$, so that $\mathfrak{A}(E \cap D)=$ 
$E+D \supseteq E \cap D$. Therefore $\operatorname{sig} B|W=\operatorname{sig} B| \mathfrak{Q}(E \cap D)=\operatorname{sig} B \mid E \oplus D$, which is equal, since $E$ and $D$ are orthogonal, to $\operatorname{sig} B|E+\operatorname{sig} B| D$.

Since $J \subseteq \operatorname{ker} B|E, \operatorname{sig} B| E=\operatorname{sig} B \mid \operatorname{ker} v$, and $S$, preserving orientation on $N$, also preserves intersection numbers, so that $\operatorname{sig} B|E=\operatorname{sig} B|$ ker $u$.

Finally, $D=\mathbb{Q} \operatorname{Ker} v \cap \mathbb{Q}$ ker $u=u^{-1}$ im $i \cap v^{-1}$ im $j=K$; since $\rho$ is induced from $-B \mid K$, by dividing out by part of the kernel, $B \mid D=\operatorname{sig}-\rho$. Therefore

$$
\sigma(T, M)=2 \operatorname{sig} B \mid \operatorname{ker} u-\operatorname{sig} \rho=2 \sigma(S ; Y, N)-\operatorname{sig} \rho .
$$

Note. Since $\sigma(T, M)$ and signature $\rho$ are determined by the embedding of $\partial N$ in $\partial Y, \sigma(S ; Y, N)$ does not depend on $N$, but only on $\partial N$, and will be denoted $\sigma(S ; Y, \partial N)$.

Corollary. Suppose $M_{0}$ and $M_{1}$ are two characteristic submanifolds of $\left(T, X^{4 k}\right)$ where $T$ reverses orientation and $X$ is closed. Then

$$
\sigma M_{0}-\sigma M_{1}=2 \sigma\left(T \times 1 ; X \times I, M_{0}-M_{1}\right)-\operatorname{sig} \rho .
$$

In particular, when $H_{2 k} X=0, \sigma M_{0}-\sigma M_{1}=2 \sigma\left(T \times 1 ; X \times I, M_{0}-M_{1}\right)$ [1] and when $X$ is a homotopy sphere, this is a multiple of 16 since the bilinear forms are even and unimodular. However, in general $\sigma M_{0}-\sigma M_{1}$ need not be even; here is an example where $\sigma M_{0}=0$ and $\sigma M_{1}=1$.

Let $X=S^{2 k} \times S^{2 k}$ and $T=A \times 1$ where $A$ is the antipodal map.

Let $M_{0}=S^{2 k-1} \times S^{2 k}$; a characteristic submanifold of $M_{0}$ is $W_{0}=$ $S^{2 k-2} \times S^{2 k}$ and since $H_{2 k-1} W_{0}=0, \sigma M_{0}=0$.

Let $M_{1}=\left\{(x, y) \in S^{2 k} \times S^{2 k} \mid x \cdot y \cdots 0\right\}$. Then $p: M_{1} \rightarrow S^{2 k}$ (projection on the first factor) is a fibre bundle with fibre $S^{2 k-1}$. $S^{2 k}$ has a characteristic submanifold $S$ dividing it into two discs, $D_{+}$and $D_{-}$. Then $W_{1}=p^{-1} S$ is a characteristic submanifold of $M_{1}$, dividing it into $p^{-1} D_{+} \cup p^{-1} D_{-}$. Since the bundle is trivial when restricted to $S, H_{2 k-1} W_{1}=Q \oplus Q$, where one generator $F$ is represented by the fibre, and the other, $C$, is represented by the cross-section $S^{2 k-1} \rightarrow M_{1}, x \rightarrow(x, a(x))$ where $a\left(0, x_{1}, \ldots, x_{2 k}\right)=$ $\left(0,-x_{2}, x_{1}, \ldots,-x_{2 k}, x_{2 k-1}\right)$. Now $T C=C$ since $\alpha \sim A \alpha$, and $T F=-F$. $p^{-1} D_{+}=D_{+} \times S^{2 k-1}$ and the map $H_{2 k-1} W_{1} \rightarrow H_{2 k-1} p^{-1} D_{+}$is determined by $C \rightarrow F$ and $F \rightarrow F$. Therefore ker $I_{2 k-1} W_{1} \rightarrow H_{2 k-1} p^{-1} D_{+}-Q$ and a generator is $C-F$. Since

$$
(C-F) \cdot T(C-F)=(C-F) \cdot(C+F)=2>0,
$$

$\sigma M_{1}=1$.

In this example $L=Q \oplus Q=H_{2 k} \partial(X \times I)$ and $\operatorname{sig} \rho=1 ; \sigma(T \times 1)=0$. 
Proposition. If $T$ is an orientation reversing involution on a manifold $X^{4 k}$ with boundary, then given $\left(M_{0}, \partial M_{0}\right)$ a characteristic submanifold of $(X, \partial X)$ where $\partial M_{0}$ divides $\partial X$ into $P \cup T P$, then there is a characteristic submanifold $\left(M_{1}, \partial M_{1}\right)$ with $\sigma M_{1}=\sigma M_{0}$, such that $\partial M_{1}=\partial M_{0}, H_{r}\left(X, A_{1} ; Z\right)$ $=0$ for $r<2 k$ and $H_{r}\left(A_{1}, P ; Q\right) \rightarrow H_{r}(X, P ; Q)$ is a monomorphism for $r \leq 2 k$.

The proof is by equivariant handle exchange between $A$ and $B$ to make the pair $(A, M) 2 k-1$ connected.

Suppose inductively that $H_{p}\left(A_{0}, M_{0} ; Z\right)=0$ for all $p<$ some fixed $r \leq$ $2 k$. Then the Hurewicz map $\pi_{r}\left(A_{0}, M_{0}\right) \rightarrow H_{r}\left(A_{0}, M_{0} ; Z\right)$ is surjective. Let $\left\{x_{1}, \ldots, x_{n}\right\} \subset \pi_{r}\left(A_{0}, M_{0}\right)$ represent a basis for $H_{r}\left(A_{0}, M_{0} ; Z\right)$. If $r<2 k, x_{1}$ can be represented by an embedding $D^{r}, S^{r-1} \subset A_{0}, M_{0}$ which is disjoint from $T x_{1}$ [8]. Let $H$ be a tubular neighborhood of $D^{r}, S^{r-1}$ in $A_{0}, M_{0}$, so that $H=D^{r} \times D^{4 k-r}$ and $H \cap M_{0}=S^{r-1} \times D^{4 k-r}$, which is disjoint from $T H$; and let $A_{1}, B_{1}$ and $M_{1}$ be the manifolds resulting from exchanging $H$ and $T H$, that is $A_{1}=A_{0}-H \cup T H, B_{1}=B_{0} \cup H-T H$ and

$$
\begin{aligned}
M_{1}= & M_{0}-\left(S^{r-1} \times D^{4 k-r}\right) \cup\left(D^{r} \times S^{4 k-r-1}\right) \\
& -T\left(S^{r-1} \times D^{4 k-r}\right) \cup T\left(D^{r} \times S^{4 k-r-1}\right) .
\end{aligned}
$$

Now $\left(A_{0}, M_{0}\right)$ is equivariantly cobordant to $\left(A_{1}, M_{1}\right)$ in $X \times I$; in particular let $K$ be a tubular neighborhood of $\left(D^{r}, S^{r-1}\right)$ in $\left(A_{0} \times I, M_{0} \times 1\right)$, and set

$$
\begin{aligned}
& U=A_{0} \times I-K \cup T K, \\
& V=(T \times 1) U=B_{0} \times I \cup K-T K \text { and } N=U \cap V .
\end{aligned}
$$

Then clearly $N$ is a characteristic submanifold of $X \times I$ and $(U, N)$ is a cobordism between $\left(A_{0}, M_{0}\right)$ and $\left(A_{1}, M_{1}\right)$.

Then $H_{r}\left(A_{1}, M_{1} ; Z\right)$ is generated by $\left\{x_{2}, \ldots, x_{n}\right\}$ and $H_{*}\left(A_{1}, M_{1} ; Z\right)=$ $H_{*}\left(A_{0}, M_{0} ; Z\right)$ in dimensions $<r$. Since $N$ is the trace of surgery on $x_{1}$ and $T x_{1}, H_{2 k}\left(N, M_{0} ; Z\right)=0$ and $H_{2 k} M_{0} \rightarrow H_{2 k} N$ is an epimorphism. Since $M_{0} C$ $\partial N$ and intersection numbers are 0 on boundaries, $\sigma M_{0}-\sigma M_{1}=\operatorname{sig} B \mid H_{2 k} N$ $[4],[5]=0$.

When $r=2 k, x_{1}$ can still be represented by an embedding of $D^{2 k}, S^{2 k-1}$ $\rightarrow A_{0}, M_{0}\left[7, \mathrm{p}\right.$. 39] disjointly from $T x_{1}$. If $x_{1}$ has infinite order in $H_{2 k}\left(A_{0}, M_{0} ; Z\right)$, surgery on $x_{1}$ will reduce the rank of $H_{2 k}\left(A_{0}, M_{0} ; Q\right)$ if and only if $\partial T x \neq 0$ in $H_{2 k-1}\left(A_{0}, P ; Q\right)$. In this case $H_{2 k} M_{0}$ is isomorphic to $H_{2 k} \mathrm{~N}$, because in the exact sequence

$$
0 \rightarrow H_{2 k} M_{0} \rightarrow H_{2 k} N \rightarrow Q x \oplus Q T x \stackrel{\partial}{\longrightarrow} H_{2 k-1} M_{0},
$$


$\partial$ is a monomorphism since the composition

$$
Q x \oplus Q T x \rightarrow H_{2 k-1} M_{0} \rightarrow H_{2 k-1} A_{0}, P \oplus H_{2 k-1} B_{0}, T P
$$

sends $(a x, b T x)$ into $(b \partial T x, a \partial x)$. Therefore, as before, $\sigma M_{0}=\sigma M_{1}$.

We can assume, then, that $M$ is a characteristic submanifold with $H_{*}(A, M ; Z)=0$ in dimensions $<2 k$, and for which the composition

$$
H_{2 k} A, M \rightarrow H_{2 k-1} M \stackrel{T}{\rightarrow} H_{2 k-1} M \rightarrow H_{2 k-1} A, P
$$

is 0 , that is, since $T$ is an isomorphism, that the maps

$$
H_{2 k} A, M \rightarrow H_{2 k-1} B, T P \text { and } H_{2 k} B, M \rightarrow H_{2 k-1} A, P
$$

are 0 . It follows that in the Mayer-Vietoris sequence

$$
\begin{gathered}
H_{2 k} X, P \rightarrow H_{2 k-1} M \rightarrow H_{2: k-1} A, P \oplus H_{2 k-1} B \rightarrow H_{2 k-1} X, P, \\
\quad \operatorname{im}\left(H_{2 k} X, P \rightarrow H_{2 k-1} M\right)=\operatorname{ker}\left(H_{2 k-1} M \rightarrow H_{2 k-1} B\right) .
\end{gathered}
$$

Taking duals we see that $\operatorname{im} i=\operatorname{im} j$

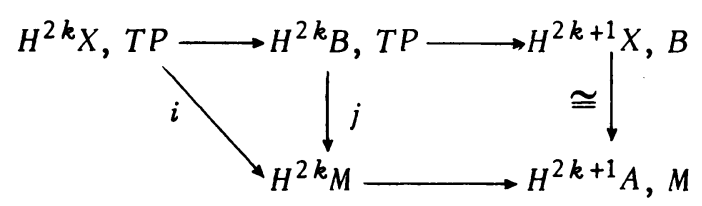

so that $h$ is an epimorphism, from which it follows that $H_{2 k}(A, P) \rightarrow$ $H_{2 k}(X, P)$ is a monomorphism. From the 5 lemma $H_{*}(A, P) \rightarrow H_{*}(X, P)$ is a monomorphism in all dimensions $\leq 2 k$.

Theorem 2. Let $T$ be an orientation reversing involution of $X^{4 k}$ where $X$ is a simply connected closed manifold and $k>1$. Let $C$ be a (vector) subspace of $H_{2 k}(X ; Q)$. Then there is a characteristic submanifold $M$ dividing $X$ into $A \cup B$ with $H_{2 k} A=C$, if and only if $C=\mathbb{Q}_{C}$, that is, since $B$ is nonsingular, if and only if $C \cdot T C=0$ and $\operatorname{dim} C=\frac{1}{2} \operatorname{dim} H_{2 k} X$.

Proof. $\Leftarrow$ Clearly if $C=H_{2 k} A, C \cdot T C=0$. Since the Alexander dual of $\operatorname{im}\left(H_{2 k} A \rightarrow H_{2 k} X\right)$ is $\operatorname{im}\left(H^{2 k} X, B \rightarrow H^{2 k} X\right)$ which is isomorphic to $\operatorname{im}\left(H_{2 k} X \rightarrow H_{2 k} X, A\right)$, and since by exactness,

$\operatorname{dim} \operatorname{im}\left(H_{2 k} A \rightarrow H_{2 k} X\right)+\operatorname{dim} \operatorname{im}\left(H_{2 k} X \rightarrow H_{2 k} X, A\right)=\operatorname{dim} H_{2 k} X$,

$2 \operatorname{dim} C=\operatorname{dim} H_{2 k} X$.

$\Rightarrow$ Suppose $f$ is an immersion: $M^{2 k} \rightarrow X$ with $f_{*}[M]=x \in H_{2 k} X$. Then there is an immersion $g$ of $M+M$, approximating $f+f$, with $g$ disjoint from 
$T g$, because if $p$ is the projection: $X \rightarrow X / T$, then the self-intersection number of $p_{*} 2 x$ will have no component in the generator of $\pi_{1} X / T$, so that $p_{*} 2 x$ can be represented without any intersections arising from intersections of $x$ and $T x[7$, p. 45]. We can then represent a basis for $C$ by immersed manifolds $M_{i}$ each disjoint from all $T M_{j}[6]$. Since $X$ is 1 -connected, the image of each $M_{i}$ can be assumed 1-connected. Let $U$ be a regular neighborhood of the union of the $M_{i}$ disjoint from $T U . X-U-T U$ has a closed characteristic submanifold $M$ dividing it into $Y \cup T Y$, where $\partial Y=\partial U$, which can be chosen so that $H_{r} Y, M=0, r<2 k$, and $H_{r}(Y, \partial U) \rightarrow H_{r}(Y \cup T Y, \partial U)$ is a monomorphism for $r \leq 2 k$. Set $A=U \cup Y$.

Now image $H_{2 k} A \rightarrow H_{2 k} X=C$ because image $H_{2 k} A \rightarrow H_{2 k} X$ is contained in its annihilator under $B$ and contains $C$ which equals $\mathbb{Q} C$. Therefore it remains to show that $H_{2 k} A \rightarrow H_{2 k} X$ is a monomorphism.

Now $a$ is a monomorphism, hence so are $b$ and $c$. Thus ker $d \subset e(\operatorname{ker} f)$; but since $g$ is a monomorphism, $\operatorname{ker} f \subset \operatorname{ker} e$; therefore $\operatorname{ker} d=0 . h$ is a monomorphism because

$$
\operatorname{ker} h=\operatorname{im}\left(H_{2 k+1} X, X-T U \rightarrow H_{2 k} X-T U\right)
$$

and $H_{2 k+1} X, X-T U=H^{2 k-1} T U$ which is 0 since each $H_{1} M=0$. Therefore hd is a monomorphism: $H_{2 k} A \rightarrow H_{2 k} X$.

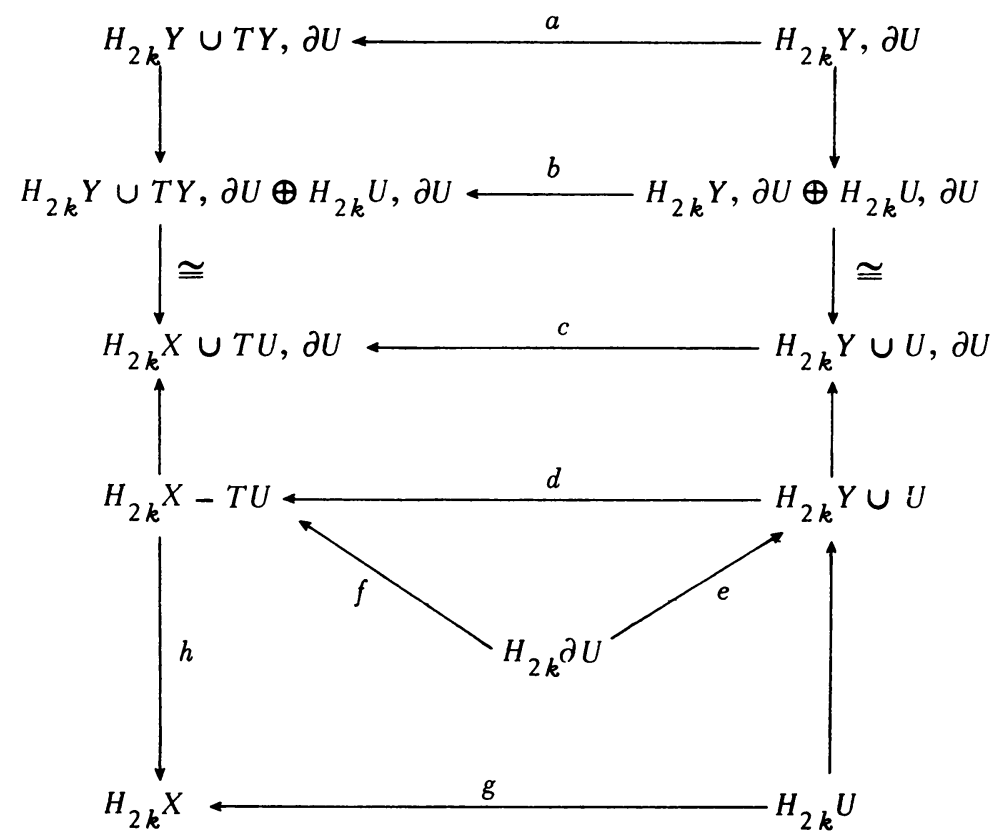

As an application we have 
Theorem 3. Let $T$ be an orientation reversing involution of $X^{4 k}$ where $X$ is a simply connected closed manifold and $k>1$. Then there is a characteristic submanifold $M_{0}$ of $X$ such that given $|r| \leq n=\operatorname{dim} H_{2 k} X$, there is another characteristic submanifold of $M_{1}$ of $X$ for which

$$
\operatorname{sig} \rho\left(M_{0}, M_{1}\right)=\sigma\left(M_{0}\right)-\sigma\left(M_{1}\right)-2 \sigma\left(X \times T_{1} M_{0}-M_{1}\right)=r .
$$

Proof. First we find a subspace $C$ of $H_{2 k} X$ for which $C \oplus T C=H_{2 k} X$ and $C \cdot T C=0$, then $C$ can be realized as $H_{2 k}(A)$ (Theorem 2); set $M_{0}=\partial A$. Let $V=\{x: x=T x\}$ and $W=\{x: x=-T x\} \subset H_{2 k} X$, then $H_{2 k} X=V \oplus W$, but since $T$ has Lefschetz number 0 , and reverses orientation, trace $T: H_{2 k} X$ $\rightarrow H_{2 k} X=0$, so that $\operatorname{dim} Y=\operatorname{dim} W=1 / 2 \operatorname{dim} H_{2 k} X$, and $V=\mathfrak{Q} V$ and $W=\mathbb{Q}_{W}$. Therefore $H_{2 k} X$ has a symplectic basis $\left\{v_{i}, w_{i}: i=1, \ldots, n / 2\right\}$, that is, $v_{i} \cdot v_{j}=0=w_{i} \cdot w_{j}$ for all $i$ and $j$ and $v_{i} \cdot w_{j} \neq 0$ only if $i=j$ and $v_{i} \cdot w_{i}$ $=1$, with $T v_{i}=v_{i}$ and $T w_{i}=-w_{i}$. Let $C$ be the space generated by $\left\{v_{i}+w_{i}: i=1, \ldots, n / 2\right\}$; then $C \cap T C=0, C+T C=H_{2 k} X$ and $C \cdot T C=0$.

Given $n / 2$ pairs of rational numbers $\left(\alpha_{i}, \beta_{i}\right)$ with each $\alpha_{i}<\beta_{i}$, let $C_{1}$ be the subspace of $H_{2 k} X$ generated by $\left\{\alpha_{i} v_{i}+\beta_{i} w_{i}\right\}$; like $C, C_{1}$ can be realized by $A_{;}$let $M_{1}=\partial A_{1}$. We wish to choose $\left\{\alpha_{i}, \beta_{i}\right\}$ so that $\operatorname{sig} \rho\left(M_{0}, M_{1}\right)$ $=r$.

Now $\rho$ is defined on $\left(C+C_{1}\right) \cap T\left(C+C_{1}\right)$ which is $H_{2 k} X$. Let $X_{i}$ denote the subspace of $H_{2 k} X$ generated by $v_{i}$ and $w_{i}$; then the family of $X_{i}$ 's are mutually orthogonal under $B$, hence also under $\rho$, since

$$
\rho\left(c+c_{1}, c^{\prime}+c_{1}^{\prime}\right)=B\left(c, c^{\prime}\right)-B\left(c_{1}, c_{1}^{\prime}\right) .
$$

Consequently sig $\rho=\Sigma \operatorname{sig} \rho \mid X_{i}$. But

$$
\rho\left|X_{i}=\right| \begin{array}{cc}
2 \beta_{i} /\left(\beta_{i}-\alpha_{i}\right) & 0 \\
0 & 2 \alpha_{i} /\left(\beta_{i}-\alpha_{i}\right)
\end{array} \mid ;
$$

since $\alpha_{i}<\beta_{i}, \operatorname{sig} \rho \mid X_{i}=\operatorname{sign} \beta_{i}+\operatorname{sign} \alpha_{i}$.

Clearly then $\alpha_{i}$ and $\beta_{i}$ can be chosen so that $\operatorname{sig} \rho \mid X_{i}$ is $2,1,0,-1$ or -2 . It follows that $\operatorname{sig} \rho$ can be any number between $-n$ and. $n$.

Since the maximum dimension, for all $M_{0}$ and $M_{1}$ of the space $L$ on which $\rho$ is defined, is $n, \rho\left(M_{0}, M_{1}\right)$ can assume all possible values.

\section{BIBLIOGRAPHY}

1. I. Berstein and G. R. Livesay, Non-unique desuspension of involutions, Invent. Math. 6 (1968), 56-66. MR 38 \#2793.

2. W. Browder and G. R. Livesay, Fixed point free involutions on homotopy 
spheres, Bull. Amer. Math. Soc. 73 (1967), 242-245. MR 34 \#6781.

3. W. Browder and G. R. Livesay, Fixed point free involutions on homotopy spheres, Tôhoku Math. J. (2) 25 (1973), 69-87. MR 47 \#9610.

4. S. López de Medrano, Involutions on manifolds, Ergebnisse der Mathematik und ihrer Grenzgebiete, Band 59, Springer-Verlag, New York, 1971. MR 45 \#7747.

5. E. Stone, Involutions on manifolds, Ph.D. Thesis, Cornell University, Ithaca, N. Y., 1972.

6. R. Thom, Quelques propriétés globales des variétés différentiables, Comment. Math. Helv. 28 (1954), 17-86. MR 15, 890.

7. C. T. C. Wall, Surgery on compact manifolds, Academic Press, New York, 1970.

8. H. Whitney, Differentiable manifolds, Ann. of Math. 37 (1936), 645-680.

DEPARTMENT OF MATHEMATICS, MICHIGAN STATE UNIVERSITY, EAST LANSING, MICHIGAN 48823 